\title{
The connected and leading disconnected diagrams of the hadronic light-by-light contribution to muon
}

$g-2$

\section{Luchang Jin*}

Physics Department, Columbia University, New York, New York 10027, USA

E-mail: ljin. luchang@gmail.com

\section{Thomas Blum}

Physics Department, University of Connecticut, Storrs, Connecticut 06269-3046, USA

RIKEN BNL Research Center, Brookhaven National Laboratory, Upton, New York 11973, USA

\section{Norman Christ}

Physics Department, Columbia University, New York, New York 10027, USA

\section{Masashi Hayakawa}

Department of Physics, Nagoya University, Nagoya 464-8602, Japan

Nishina Center, RIKEN, Wako, Saitama 351-0198, Japan

\section{Taku Izubuchi}

Physics Department, Brookhaven National Laboratory, Upton, New York 11973, USA

RIKEN BNL Research Center, Brookhaven National Laboratory, Upton, New York 11973, USA

\section{Chulwoo Jung}

Physics Department, Brookhaven National Laboratory, Upton, New York 11973, USA

\section{Christoph Lehner}

Physics Department, Brookhaven National Laboratory, Upton, New York 11973, USA

\begin{abstract}
We report our recent lattice calculation of hadronic light-by-light contribution to muon $g-2$ using our recently developed moment method. The connected diagrams and the leading disconnected diagrams are included. The calculation is performed on a $48^{3} \times 96$ lattice with physical pion mass and $5.5 \mathrm{fm}$ box size. We expect sizable finite volume and finite lattice spacing corrections to the results of these calculations which will be estimated in calculations to be carried out over the next 1-2 years.
\end{abstract}

34th annual International Symposium on Lattice Field Theory

24-30 July 2016

University of Southampton, UK

\footnotetext{
* Speaker.
} 


\section{Introduction}

The anomalous magnetic moment of muon, $a_{\mu}$, can be measured very precisely by experiments. The dimensionless number, $a_{\mu}$, is defined through the following relation:

$$
\vec{\mu}=2\left(1+a_{\mu}\right) \frac{-e}{2 m_{\mu}} \vec{s}
$$

where $\vec{\mu}$ is the magnetic moment of muon and $\vec{s}$ is its spin. The past experiment, BNL E821 [1], has obtained $a_{\mu}^{\exp }=11659208.0(6.3) \times 10^{-10}$. However, this value is larger than the standard model prediction, whose uncertainty is estimated to be around $5.9 \times 10^{-10}$, by three standard deviations [2]. Before one can declare the discovery of physics beyond the standard model, both the experimental value and the theoretical prediction need to be improved. Therefore, much more accurate experiments, Fermilab E989 [3] and J-PARC E34 [4], are under active preparation. The experimental uncertainties are expected be reduced to $1 / 4$ of its current value. On the other hand, in order to improve the accuracy of the standard model prediction, one need to address two kinds of diagrams shown in Fig. 1, namely the hadronic vacuum polarization (HVP) diagram and the hadronic light-by-light (HLbL) diagram, which are the major sources of uncertainty in the current theoretical prediction.

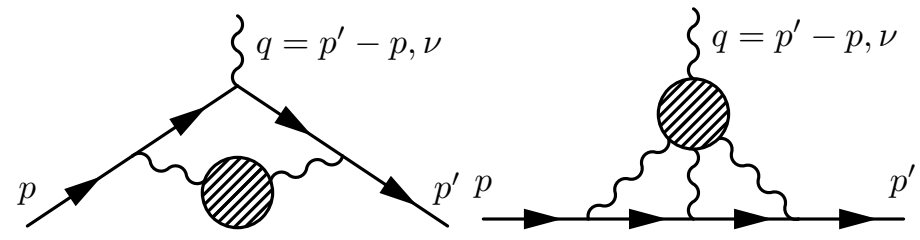

Figure 1: (Left) Hadronic vacuum polarization (HVP). (Right) Hadronic light-by-light (HLbL).

In this proceeding, we will discuss the lattice calculation of the connected and leading disconnected hadronic light-by-light amplitude. Previously this quantity has only been calculated using models [5]. Attempts using lattice QCD were begun by T. Blum, M. Hayakawa, and T. Izubuchi more than 6 years ago $[6,7]$. We have improved the methodology dramatically, as described in Refs $[8,9,10]$, which leads to a reduction in statistical errors by more than an order of magnitude, and includes the leading disconnected-diagram contribution for the first time. Since much of the material that was presented at LATTICE 2016 has now appeared in a recent paper [10], this proceeding, after a short summary of the connected and leading disconnected diagram calculation, is devoted to an expanded discussion of a topic that was only briefly presented during the conference: the large separation behaviour of the four-point-function within the light-by-light diagram.

\section{Lattice calculation setup and results}

We start the discussion by repeating our final moment-method formula for evaluating the connected light-by-light contribution to $a_{\mu}^{\mathrm{cHLbL}}$ obtained in Refs $[8,9,10]$.

$$
a_{\mu}^{\mathrm{cHLbL}} \frac{\left(\sigma_{s^{\prime} s}\right)_{i}}{2 m_{\mu}}=\sum_{r, \tilde{z}} \mathfrak{Z}\left(\frac{r}{2},-\frac{r}{2}, \tilde{z}\right) \sum_{\tilde{x}_{\mathrm{op}}} \frac{1}{2} \varepsilon_{i j k}\left(\tilde{x}_{\mathrm{op}}\right)_{j} \cdot i \bar{u}_{s^{\prime}}(\overrightarrow{0}) \mathscr{F}_{k}^{C}\left(\frac{r}{2},-\frac{r}{2}, \tilde{z}, \tilde{x}_{\mathrm{op}}\right) u_{s}(\overrightarrow{0}),
$$

where $\left(\sigma_{s^{\prime} s}\right)_{i}=\bar{u}_{s^{\prime}}(\overrightarrow{0}) \Sigma_{i} u_{s}(\overrightarrow{0})$ are the conventional Pauli matrices, $\Sigma_{k}=\frac{1}{4 i} \varepsilon_{i j k}\left[\gamma_{i}, \gamma_{j}\right]$, and the weight function " $\mathfrak{Z}$ " is defined in Ref. [8, 10], but could be replaced by 1 without hurting correctness. The integration variables are related to the coordinates in the first three diagrams in Figure 2 by the 
following equations: $r=x-y, \tilde{z}=z-(x+y) / 2, \tilde{x}_{\mathrm{op}}=x_{\mathrm{op}}-(x+y) / 2$. The function $\mathscr{F}_{v}^{C}\left(x, y, z, x_{\mathrm{op}}\right)$ represent the Feynman amplitude; an explicit expression is given in Ref [10].

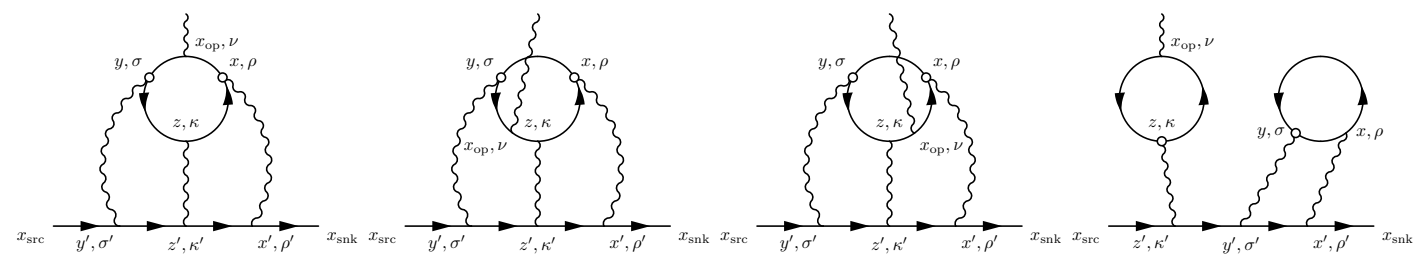

Figure 2: The first three diagrams show the three different ways of inserting the external photon when the vertices $x$ and $y$ are fixed. The forth diagram is the leading order disconnected diagram, the only type of disconnected diagrams that survies in SU(3) limit, other disconnected diagrams are not included in the calculation yet. They are listed in Ref. [10]. For each of these four diagrams, there are five other possible permutations of the connections between the three internal photons and the muon line that are not shown.

We perform the sum over $r=x-y$ by sampling a few values on each QCD configuration and use point source propagators at $x$ and $y$. All other coordinates are summed over completely on the lattice using the point source propagators and the sequential source propagators. Since $x$ and $y$ are connected by quark lines, it can be expected that the major contribution to $g-2$ comes from the region where $|r|$ is small. We accommodate this by using importance sampling. We even perform a complete sum, up to discrete symmetries, in the region where $|r| \leqslant r_{\max } . r_{\max }$ is chosen to be 5 in lattice units in our numerical simulations.

For the disconnected diagrams in Figure 2, the moment method can still be applied.

$$
a_{\mu}^{\mathrm{dHLbL}} \frac{\left(\sigma_{s^{\prime}, s}\right)_{i}}{2 m_{\mu}}=\sum_{r, \tilde{x}} \sum_{\tilde{x}_{\mathrm{op}}} \frac{1}{2} \varepsilon_{i, j, k}\left(\tilde{x}_{\mathrm{op}}\right)_{j} \cdot i \bar{u}_{s^{\prime}}(\overrightarrow{0}) \mathscr{F}_{k}^{D}\left(\tilde{x}, 0, r, r+\tilde{x}_{\mathrm{op}}\right) u_{s}(\overrightarrow{0}) .
$$

The integration variables are related to the coordinates in the forth diagram in Figure 2 by the following equations: $r=z-y, \tilde{x}=x-y, \tilde{x}_{\mathrm{op}}=x_{\mathrm{op}}-z$. Just like the connected diagram calculation, the sum over $r$ is performed by randomly sampling the $(z, y)$ point-pairs. In order to increase statistics, we compute point source propagators for 1024 randomly (not uniformly, see Ref [10]) chosen points and use all possible $1024^{2}$ combinations as the sample of point-pairs. The amplitude $\mathscr{F}_{v}^{D}\left(x, y, z, x_{\mathrm{op}}\right)$ is given by:

$$
\mathscr{F}_{v}^{D}\left(x, y, z, x_{\mathrm{op}}\right)=(-i e)^{6} \mathscr{G}_{\rho, \sigma, \kappa}(x, y, z)\left\langle\frac{1}{2} \Pi_{v, \kappa}\left(x_{\mathrm{op}}, z\right)\left[\Pi_{\rho, \sigma}(x, y)-\Pi_{\rho, \sigma}^{\mathrm{avg}}(x-y)\right]\right\rangle_{\mathrm{QCD}}
$$

The function $\mathscr{G}_{\rho, \sigma, \kappa}(x, y, z)$ represents the photon and muon part of the diagram [10]. The function $\Pi_{\rho, \sigma}(x, y)$ is given by the following expression:

$$
\Pi_{\rho, \sigma}(x, y)=-\sum_{q}\left(e_{q} / e\right)^{2} \operatorname{Tr}\left[\gamma_{\rho} S_{q}(x, y) \gamma_{\sigma} S_{q}(y, x)\right] .
$$

The subtraction term in Eq. (2.3) does not contribute to the central value [10]. We only perform this subtraction in our lattice calculation as a noise reduction technique, and we are free to choose the subtraction term $\Pi^{\text {avg }}$ as long as it is a pre-determined constant in the ensemble average process. Note that a similar subtraction would be essential if the moment method is not applied [11].

The calculations were performed on the $48^{3} \times 96$ physical-pion-mass ensemble [12] generated by the RBC and UKQCD collaborations. We have performed the calculation it on 65 configurations each separated by $20 \mathrm{MD}$ units. Based on our strategy stated above, we sample point-pairs to perform the sum over $r$. In Figure 3, we plot the histograms based on those point-pairs. 
The sum of the contributions from all separations gives the result: $a_{\mu}^{\mathrm{cHLbL}}=(11.60 \pm 0.96) \times$ $10^{-10}$, and $a_{\mu}^{\mathrm{dHLbL}}=(-6.25 \pm 0.80) \times 10^{-10}$. Thus

$$
a_{\mu}^{\mathrm{HLbL}}=(5.35 \pm 1.35) \times 10^{-10} .
$$

The errors are statistical only. It might come as a surprise that the disconnected diagram contribution is quite large. We will show in the next section that this result is more or less expected.
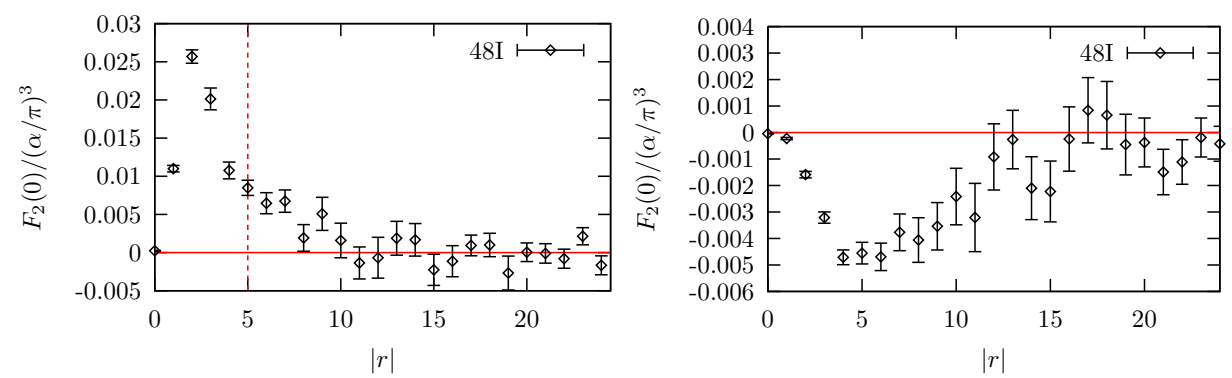

Figure 3: Histograms of the contribution to $F_{2}$ from different separations $|r|$. The $i$ th bin includes contributions from the region where $i-1<|r| \leqslant i$. The sum of all these bins gives the final result for $F_{2}$. Left: cHLbL contribtion and $r=x-y$. Right: dHLbL contribution and $r=z-y$.

\section{Connections between the connected and disconnected diagrams}

\subsection{The $\pi^{0}$ exchange diagram}

We know that $\pi^{0}$ is the lightest particle in QCD, because of the spontaneous chiral symmetry breaking and Goldstone mechanism. For two points $x, y$ separated by long distance

$$
\begin{aligned}
& \left\langle\left(\bar{u} \gamma_{5} u-\bar{d} \gamma_{5} d\right)(x)\left(\bar{u} \gamma_{5} u-\bar{d} \gamma_{5} d\right)(y)\right\rangle \sim e^{-m_{\pi}|x-y|}, \\
& \left\langle\left(\bar{u} \gamma_{5} u+\bar{d} \gamma_{5} d\right)(x)\left(\bar{u} \gamma_{5} u+\bar{d} \gamma_{5} d\right)(y)\right\rangle \sim e^{-m_{\eta}|x-y|} .
\end{aligned}
$$

Assuming perfect isospin symmetry, the above relations imply:

$$
\begin{aligned}
\left\langle\bar{u} \gamma_{5} u(x)\left(\bar{u} \gamma_{5} u-\bar{d} \gamma_{5} d\right)(y)\right\rangle & \sim e^{-m_{\pi}|x-y|}, \\
\left\langle\bar{u} \gamma_{5} u(x) \bar{d} \gamma_{5} d(y)\right\rangle+\frac{1}{2}\left\langle\bar{u} \gamma_{5} u(x)\left(\bar{u} \gamma_{5} u-\bar{d} \gamma_{5} d\right)(y)\right\rangle & \sim e^{-m_{\eta}|x-y|} .
\end{aligned}
$$

This tells us a relationship between the connected diagram and the disconnected diagram:

$$
\left\langle\bar{u} \gamma_{5} u(x) \bar{d} \gamma_{5} d(y)\right\rangle=-\frac{1}{2}\left\langle\bar{u} \gamma_{5} u(x)\left(\bar{u} \gamma_{5} u-\bar{d} \gamma_{5} d\right)(y)\right\rangle\left[1+\mathscr{O}\left(e^{-\left(m_{\eta}-m_{\pi}\right)|x-y|}\right)\right]
$$

where the left hand side represents only disconnected diagrams, while the right hand side represents only connected diagrams.
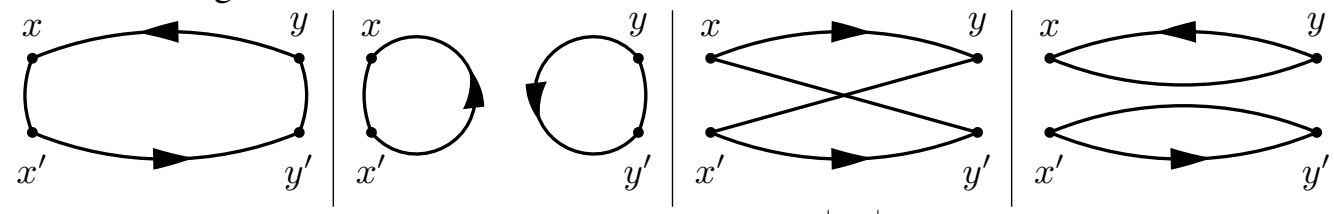

Figure 4: (First) Connected diagram $C\left(x, x^{\prime}, y, y^{\prime}\right) \sim e^{-m_{\pi}|x-y|}$. (Second) Disconnected diagram $D\left(x, x^{\prime}, y, y^{\prime}\right) \sim e^{-m_{\pi}|x-y|}$. (Third) Suppressed connected diagram $\mathscr{O}\left(e^{-2 m_{\pi}|x-y|}\right)$. (Fourth) Suppressed disconnected diagram $\mathscr{O}\left(e^{-2 m_{\pi}|x-y|}\right)$. The polarization indexes are always associated with the coordinates, and are thus omitted.

In the hadronic light-by-light process, we have a hadronic four point function. Let's denote the four points to be $x, x^{\prime}, y, y^{\prime}$. In the region where $x$ is far separated from $y$, but $x^{\prime}$ is close to $x$ and $y^{\prime}$ is close to $y$, we know that the four point function should be dominated by the $\pi^{0}$ exchange process. In order to deal with this hadronic four point function, we first consider a four-point function in 
terms of the non-singlet current operator $V_{\mu}(x)=\bar{u} \gamma_{\mu} u(x)-\bar{d} \gamma_{\mu} d(x)$. It can be expressed in terms of the diagrams shown in Figure 4.

$$
\begin{gathered}
\left\langle V_{\mu}(x) V_{v}\left(x^{\prime}\right) V_{\rho}(y) V_{\sigma}\left(y^{\prime}\right)\right\rangle=2\left[C\left(x, x^{\prime}, y, y^{\prime}\right)+C\left(x, x^{\prime}, y^{\prime}, y\right)+C\left(x^{\prime}, x, y, y^{\prime}\right)+C\left(x^{\prime}, x, y^{\prime}, y\right)\right] \\
+4 D\left(x, x^{\prime}, y, y^{\prime}\right)+\mathscr{O}\left(e^{-2 m_{\pi}|x-y|}\right) .
\end{gathered}
$$

Since the operator $V$ has isospin $I=1$, the state created by the product $V_{\mu}(x) V_{v}\left(x^{\prime}\right)$ or $V_{\rho}(y) V_{\sigma}\left(y^{\prime}\right)$ has isospin $I=0$ or $I=2$. Thus, the energy of this state is at least $2 m_{\pi}$, and the four point function must behave like $\mathscr{O}\left(e^{-2 m_{\pi}|x-y|}\right)$. When combined with Eq. (3.6), we learn that:

$$
\begin{gathered}
D\left(x, x^{\prime}, y, y^{\prime}\right)=-\frac{1}{2}\left[C\left(x, x^{\prime}, y, y^{\prime}\right)+C\left(x, x^{\prime}, y^{\prime}, y\right)+C\left(x^{\prime}, x, y, y^{\prime}\right)+C\left(x^{\prime}, x, y^{\prime}, y\right)\right] \\
+\mathscr{O}\left(e^{-2 m_{\pi}|x-y|}\right) .
\end{gathered}
$$

We can see that this is very similar to Eq. (3.5). This is because the product of the two nearby current operators $\left[\bar{u} \gamma_{\mu} u(x)\right]\left[\bar{u} \gamma_{\mu} u\left(x^{\prime}\right)\right]$ can be viewed as a non-local version of $\bar{u} \gamma_{5} u(x)$. Now, we can come back to our original four-point function with $J_{\mu}=e_{u} \bar{u} \gamma_{\mu} u(x)+e_{d} \bar{d} \gamma_{\mu} d(x)$.

$$
\begin{aligned}
& \left\langle J_{\mu}(x) J_{v}\left(x^{\prime}\right) J_{\rho}(y) J_{\sigma}\left(y^{\prime}\right)\right\rangle \\
& =\left(e_{u}^{4}+e_{d}^{4}\right)\left[C\left(x, x^{\prime}, y, y^{\prime}\right)+C\left(x, x^{\prime}, y^{\prime}, y\right)+C\left(x^{\prime}, x, y, y^{\prime}\right)+C\left(x^{\prime}, x, y^{\prime}, y\right)\right] \\
& \quad+\left(e_{u}^{2}+e_{d}^{2}\right)^{2} D\left(x, x^{\prime}, y, y^{\prime}\right)+\mathscr{O}\left(e^{-2 m_{\pi}|x-y|}\right)+\text { sub-leading disconnected diagrams. }
\end{aligned}
$$
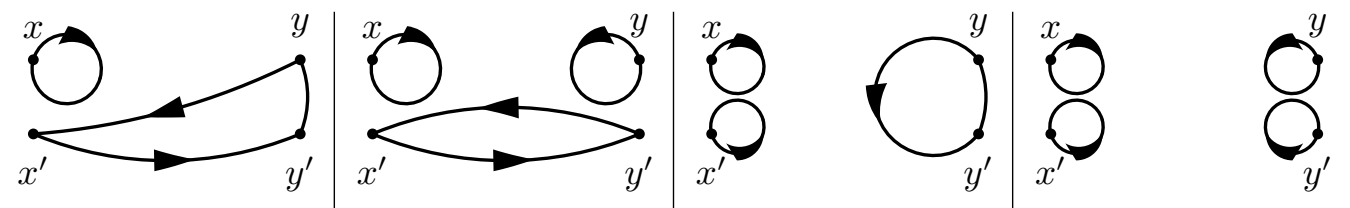

Figure 5: Various kinds of sub-leading disconnected diagrams.

Here the sub-leading disconnected diagrams refer to the diagrams vanishing in $\mathrm{SU}(3)$ limit, some of them are shown in Figure 5. These diagrams are only responsible for a small portion of the pion exchange contribution, since they only couple to $\pi^{0}$ through the disconnected diagrams in the $\pi^{0} \rightarrow \gamma \gamma$ process, and the disconnected diagrams in this process are known to be very small [13, 14]. Nevertheless, contributions from these diagrams do not affect the ratio between the leading disconnected diagrams and the connected diagrams. Combining Eq. (3.7)(3.8), we obtained that:

$$
\frac{\operatorname{dHLbL}\left(x, x^{\prime}, y, y^{\prime}\right)}{\operatorname{cHLbL}\left(x, x^{\prime}, y, y^{\prime}\right)}=\frac{\left(e_{u}^{2}+e_{d}^{2}\right)^{2}\left(-\frac{1}{2}\right)}{\left(e_{u}^{4}+e_{d}^{4}\right)}+\mathscr{O}\left(e^{-m_{\pi}|x-y|}\right)=\frac{-25}{34}+\mathscr{O}\left(e^{-m_{\pi}|x-y|}\right) .
$$

In comparison, [15], by assuming $\pi^{0}$ exchange dominance and investigating the large $N_{c}$ behavior, independently estimated that the total disconnected contribution to $\mathrm{HLbL}$ is large and negative with a ratio of disconnected to connected of $-25 / 34$.

A similar relation also appears in the hadronic vacuum polarization (HVP) calculation, which was already known in Ref [16]. since the lightest $I, J=0,1$ state has three pions, while the lightest $I, J=1,1$ state only has two pions, we know that for far separated $x$ and $y$, we have

$$
\begin{aligned}
& \left\langle\left(\bar{u} \gamma_{\mu} u-\bar{d} \gamma_{\mu} d\right)(x)\left(\bar{u} \gamma_{\mu} u-\bar{d} \gamma_{\mu} d\right)(y)\right\rangle \sim e^{-2 m_{\pi}|x-y|}, \\
& \left\langle\left(\bar{u} \gamma_{\mu} u+\bar{d} \gamma_{\mu} d\right)(x)\left(\bar{u} \gamma_{\mu} u+\bar{d} \gamma_{\mu} d\right)(y)\right\rangle \sim e^{-3 m_{\pi}|x-y|} .
\end{aligned}
$$

Following the same logic, when $x$ and $y$ are far separated:

$$
\left\langle\bar{u} \gamma_{\mu} u(x) \bar{d} \gamma_{\mu} d(y)\right\rangle \approx-\frac{1}{2}\left\langle\bar{u} \gamma_{\mu} u(x)\left(\bar{u} \gamma_{\mu} u-\bar{d} \gamma_{\mu} d\right)(y)\right\rangle\left[1+\mathscr{O}\left(e^{-m_{\pi}|x-y|}\right)\right] .
$$


With this relation, we know that the ratio between the disconnected HVP contribution and the connected HVP contribution at large $x, y$ separation is:

$$
\frac{\operatorname{dHVP}(x, y)}{\operatorname{cHVP}(x, y)}=\frac{\left(e_{u}+e_{d}\right)^{2}\left\langle\bar{u} \gamma_{\mu} u(x) \bar{d} \gamma_{\mu} d(y)\right\rangle}{\left(e_{u}^{2}+e_{d}^{2}\right)\left\langle\bar{u} \gamma_{\mu} u(x)\left(\bar{u} \gamma_{\mu} u-\bar{d} \gamma_{\mu} d\right)(y)\right\rangle}=\frac{-1}{10}+\mathscr{O}\left(e^{-m_{\pi}|x-y|}\right)
$$

\subsection{Charged pion loop contribution}

Although it is estimated that the charged pion loop does not play a significant role in the HLbL process [2], its contribution can be unambiguously identified when all four points in the hadronic four-point-function are far separated.

There are three types of diagrams which contribute through the charged pion loop : 1) the connected diagram, 2) the leading disconnected diagram, 3) the sub-leading disconnected diagrams with two quark loops, where one loop is attached to only one photon, the other loop is attached to three photons. We denote their contribution to $\mathrm{HLbL}$ without the charge factors by $C, D, D^{\prime}$. The overall contribution is:

$$
\left(e_{u}^{4}+e_{d}^{4}\right) C+\left(e_{u}^{2}+e_{d}^{2}\right)^{2} D+\left(e_{u}+e_{d}\right)\left(e_{u}^{3}+e_{d}^{3}\right) D^{\prime} \propto\left(e_{u}-e_{d}\right)^{4} .
$$

Since we study the region where the charged pion loop dominates, we expect the sum of all diagrams is proportion to the pion charge to the forth power, because there are four photon - charged pion vertices. Above relations should be true for any value of $e_{u}$ and $e_{d}$. According to this, we can derive that $D \approx \frac{3}{2} C$, and $D^{\prime} \approx-2 C$. As a result, in the region where all four points of the four-point function are far separated, the ratio between the leading disconnected diagram and the connected diagram is:

$$
\frac{\mathrm{dHVP}}{\mathrm{cHVP}}=\frac{\left(e_{u}^{2}+e_{d}^{2}\right)^{2} D}{\left(e_{u}^{4}+e_{d}^{4}\right) C} \approx \frac{-75}{-34} .
$$

One can easily apply this method to the problems discussed in the previous subsection and obtain exactly the same result. However, this arguement, relying on the assumption that the coupling is proportion to hadron charge, is not as rigorous as the method discussed above, and one cannot easily estimate the size of remainly effects like we did in the previous subsection.

\section{Conclusions and ackownlegements}

We briefly summarized the lattice calculation of the hadronic light-by-light contribution to muon anomalous magnetic moment, including the leading disconnected diagram. The calculation follows the method developed in previous work $[8,9]$, and this calculation is also described in Ref [10]. We also discussed the large separation behaviour of the four-point function within the hadronic light-by-light diagram, and obtained the theoretical ratio between the connected and the leading disconnected diagrams in different situations. We plan to a) address the discretization errors by computing on our finer, physical-pion-mass $64^{3}$ lattice with similar physical volume. b) address the finite volume effect by using the $48^{3}$ QCD ensemble inside a larger QED box or infinite volume analytic formula. and c) compute additional sub-leading disconnected diagrams.

We would like to thank our RBC and UKQCD collaborators for helpful discussions and support. We would also like to thank RBRC and BNL for BG/Q computer time. The $48^{3}$ computation is performed on Mira with ALCC allocation using BAGEL [17] library. The CPS [18] software package is also used in the calculation. The computation is performed under the ALCC Program of the US DOE on the Blue Gene/Q (BG/Q) Mira computer at the Argonne Leadership Class Facility, a DOE Office of Science Facility supported under Contract De-AC02-06CH11357. T.B is supported by U.S. DOE grant \#DE-FG02-92ER41989. N.H.C and L.C.J are supported by U.S. DOE 
grant \#DE-SC0011941. M.H is supported by Grants-in-Aid for Scientific Research \#25610053. T.I, C.J. and C.L are supported by U.S. DOE Contract \#AC-02-98CH10996(BNL). T.I. is supported in part by the Japanese Ministry of Education Grant-in-Aid, No. 26400261. CL acknowledges support through a DOE Office of Science Early Career Award.

\section{References}

[1] G. Bennett et al. In: Phys.Rev. D73 (2006), p. 072003. arXiv: hep-ex / 0602035 [hep-ex] .

[2] F. Jegerlehner and A. Nyffeler. In: Phys. Rept. 477 (2009), pp. 1-110. arXiv: 0902.3360 [hep-ph].

[3] R. M. Carey et al. In: (2009).

[4] T. Mibe. In: Chin. Phys. C34 (2010), pp. 745-748.

[5] J. Prades, E. de Rafael, and A. Vainshtein. In: (2009). arXiv: 0901.0306 [hep-ph ].

[6] M. Hayakawa et al. In: PoS LAT2005 (2006), p. 353. arXiv: hep-lat/ 0509016 [hep-lat ].

[7] T. Blum et al. In: Phys.Rev.Lett. 114.1 (2015), p. 012001. arXiv: 1407.2923 [hep-lat ].

[8] L. Jin et al. In: 12th Conference on the Intersections of Particle and Nuclear Physics (CIPANP 2015) Vail, Colorado, USA, May 19-24, 2015. 2015. arXiv: 1509.08372 [hep-lat] .

[9] T. Blum et al. In: Phys. Rev. D93.1 (2016), p. 014503. arXiv: 1510.07100 [hep-lat ].

[10] T. Blum et al. In: (2016). arXiv: 1610.04603 [hep-lat ].

[11] M. Hayakawa et al. In: PoS LATTICE2015 (2016), p. 104. arXiv: 1511.01493 [hep-lat ] .

[12] T. Blum et al. In: Phys. Rev. D93.7 (2016), p. 074505. arXiv: 1411.7017 [hep-lat ].

[13] X. Feng et al. In: Phys. Rev. Lett. 109 (2012), p. 182001. arXiv: 1206.1375 [hep-lat ].

[14] A. Gérardin, H. B. Meyer, and A. Nyffeler. In: Phys. Rev. D94.7 (2016), p. 074507. arXiv: 1607.08174 [hep-lat].

[15] J. Bijnens and J. Relefors. In: JHEP 09 (2016), p. 113. arXiv: 1608.01454 [hep-ph ].

[16] A. Francis et al. In: Phys. Rev. D88 (2013), p. 054502. arXiv: 1306.2532 [hep-lat].

[17] P. A. Boyle. In: Comput. Phys. Commun. 180 (2009), pp. 2739-2748.

[18] C. Jung. In: PoS LATTICE2013 (2014), p. 417. 